\title{
Influence of the Menstrual Cycle on Blood Markers of Muscle Damage and Inflammation Following Eccentric Exercise
}

\author{
Nuria Romero-Parra ${ }^{1,+}+\mathbb{D}$, Laura Barba-Moreno ${ }^{1, *,+}$, Beatriz Rael $^{1,+}{ }^{\mathbb{D}}$, \\ Víctor M. Alfaro-Magallanes ${ }^{1,+}$, Rocío Cupeiro ${ }^{1,+}{ }^{\mathbb{C}}$, Ángel E. Díaz ${ }^{2} \mathbb{D}$, Francisco J. Calderón ${ }^{1,+}$ \\ and Ana B. Peinado ${ }^{1, \dagger}$ \\ 1 LFE Research Group. Department of Health and Human Performance. Faculty of Physical Activity and \\ Sport Sciences. Universidad Politécnica de Madrid (UPM), 28040 Madrid, Spain; n.romero@upm.es (N.R.-P.); \\ beanad16@gmail.com (B.R.); vm.alfaro@upm.es (V.M.A.-M.); rocio.cupeiro@upm.es (R.C.); \\ franciscojavier.calderon@upm.es (F.J.C.); anabelen.peinado@upm.es (A.B.P.) \\ 2 Clinical Laboratory. National Center of Sport Medicine. Health and Sports Department, Agencia Española \\ de Protección de la Salud en el Deporte (AEPSAD), 28040 Madrid, Spain; enrique.diaz@aepsad.gob.es \\ * Correspondence: laura.barba@upm.es; Tel.: +34-910677866 \\ $+\quad$ N.R.-P., L.B.-M., B.R., V.M.A.-M., R.C., F.J.C. and A.B.P. on behalf of The IronFEMME Study Group.
}

Received: 12 February 2020; Accepted: 28 February 2020; Published: 2 March 2020

\begin{abstract}
The aim of this study was to evaluate whether the menstrual cycle and its underlying hormonal fluctuations affect muscle damage and inflammation in well-trained females following an eccentric exercise. Nineteen eumenorrheic women performed an eccentric squat-based exercise in the early follicular phase, late follicular phase and mid-luteal phase of their menstrual cycle. Sex hormones and blood markers of muscle damage and inflammation -creatine kinase, myoglobin, lactate dehydrogenase, interleukin-6, tumoral necrosis factor- $\alpha$, and $C$ reactive protein- were analyzed in each phase. No effect of menstrual cycle phase was observed $(p>0.05)$, while an interaction for interleukin-6 was shown $(p=0.047)$. Accordingly, a moderate effect size [0.68 (0.53)-0.84 (0.74)], indicated that interleukin-6 values $2 \mathrm{~h}$ post-trial $(2.07 \pm 1.26 \mathrm{pg} / \mathrm{mL})$ were likely to be higher than baseline $(1.59 \pm 0.33 \mathrm{pg} / \mathrm{mL}), 24 \mathrm{~h}(1.50 \pm 0.01 \mathrm{pg} / \mathrm{mL})$ and $48 \mathrm{~h}(1.54 \pm 0.13 \mathrm{pg} / \mathrm{mL})$ in the mid-luteal phase. Blood markers of muscle damage and inflammation were not affected by the menstrual cycle in well-trained women. The eccentric exercise barely triggered muscle damage and hence, no inflammation was observed, possibly due to participants training status. The mid-luteal phase was the only phase reflecting a possible inflammatory response in terms of interleukin-6, although further factors than sex hormones seem to be responsible for this finding.
\end{abstract}

Keywords: sex hormones; creatine kinase; inflammation; female

\section{Introduction}

The release of muscle-specific enzymes and proteins into the blood stream is one of the consequences of exercise-induced muscle damage, which is evident from isometric exercise at a long muscle length but it is predominately elicited by eccentric muscle contractions [1]. Muscles may not be accustomed to these exceeding loads which overstretch sarcomeres, and thus, sarcolemma and t-tubules are disrupted [1]. As a result, membrane permeability increases, and calcium enters the cytosol which stimulates some enzymes that may degrade contracting proteins [1]. This contributes to the release of creatine kinase (CK) in the blood stream [2], which has been widely monitored in sports medicine [3], as well as the potential release of myoglobin and lactate dehydrogenase (LDH) [2]. The acute phase of the muscle damage response is characterized by the inflammatory process in response 
to injury, which facilitates the movement of fluids and cells into the damaged tissue [4]. Essentially, inflammatory cells are involved in the clearance of debris while myogenic satellite cells promote repair and remodeling of tissues $[4,5]$. The downstream process is triggered by circulating tissue resident leukocytes, among which, neutrophils immediately arrive at the site of injury within the first 12 hours. As a consequence, the production of substances such as reactive oxygen and nitrogen species increases, as well as the secretion of pro-inflammatory cytokines such as tumoral necrosis factor- $\alpha$ (TNF- $\alpha)[4,5]$. This cytokine- TNF- $\alpha$ - enhances the release of interleukin-6 [IL-6] in response to exercise; however, IL-6 is also released by pro-inflammatory macrophages that arrived at the site of injury to propagate the inflammatory response by secreting other cytokines such as interleukin-1 or interleukin-2 [4,5]. These are the main mediators of inflammation as they attract inflammatory cells and stimulate satellite cell proliferation [4]. However, IL-6 in response to exercise also plays an anti-inflammatory role as it triggers T-regulatory lymphocyte differentiation. Within the first 24 hours, most damaged tissues are phagocytosed, and after 24 hours, anti-inflammatory macrophages and T-regulatory lymphocytes replace the pro-inflammatory cells; therefore, reducing inflammation and enhancing myoblast and differentiation, which promotes the repair of tissues [1]. The release of all these markers into the blood stream and their clearance from plasma also depends on some factors such as participants' training status, the type, intensity and duration of exercise, and the performance of subsequent bouts of exercise [2,3]. Moreover, the fact that some subjects could be high or low responders, with regards to reporting higher or lower elevations of these serum markers in response to the same stimulus, should be also considered [2]. Finally, the complexity of the female's hormonal environment due to sex hormones fluctuations throughout the menstrual cycle may be seen as an additional barrier to evaluate muscle damage and inflammation responses to exercise.

Animal studies provide some evidence of estrogens' protective effects against muscle damage [4,5]. These effects consist of playing an antioxidant role-due to their structure, estrogens facilitate the donation of hydrogens, limit peroxidation chain reactions, and therefore, contribute to membrane stability [6]. In human models, specifically in postmenopausal females, the use of estrogen-based replacement therapies has demonstrated to be beneficial to strength [7], as well as reducing muscle damage and inflammation markers after exercise [8]. However, regarding premenopausal women this effect is not clear. A possible reason could be the complexity of understanding the menstrual cycle and its hormonal fluctuations $[9,10]$. Estrogen levels vary from their nadirs at the early follicular phase (EFP) coinciding with menses, to its peak at the late follicular phase (LFP) just prior ovulation [11]. After that, estrogen levels fall but moderately rise later in the mid-luteal phase (MLP), when progesterone levels reaches its peak [11]. Thus, such fluctuations should be considered when premenopausal women are evaluated [9].

According to previous research evaluating muscle damage on premenopausal female participants, lower myoglobin or CK responses during high-estrogen phases have been observed [10,12-14]. This has been attributed to the presence of estrogen in myofibrils, which may mitigate mechanical damage to muscle fiber membranes; therefore, reducing the release of such proteins into the blood stream, and downregulating inflammation-enhancing genes [10,12-14]. In contrast, the lack of differences in muscle damage markers between phases observed in other studies $[15,16]$ have been related to the fact that estrogen concentrations may not differ enough between phases to provide protective musculoskeletal benefits, being therefore estrogen concentration during menses sufficient to offer protection against muscle damage [15]. Further possible reasons of lacking differences could be the individual variation in estrogen concentration between participants or their training status [15]. Nonetheless, most of female studies assessing exercise-induced muscle damage only evaluated one phase of the menstrual cycle due to pursuing different aims such as: comparing eumenorrheic women to oral contraceptive users $[17,18]$, comparing female to male responses [18-20], observing responses after different protocols [21] or evaluating the repeated bout effect $[22,23]$. Therefore, the most important aspect affecting the results from previous studies could be the underrepresentation of the entire menstrual cycle [11], in most cases followed by an incomplete methodology to verify the menstrual cycle phases [24]. 
On the basis of these controversial findings, the purpose of this study was to evaluate whether the menstrual cycle and the underlying hormonal changes influence blood markers of muscle damage and inflammation, following an eccentric squat-based workout. The squat exercise was chosen considering that it is a functionally relevant movement and a commonly used exercise. Hence, despite being performed in laboratory conditions it could reflect a lifelike training in well-trained females. We hypothesized that muscle damage and inflammation markers present a more pronounced response when concentrations of sex steroid hormones are lower, as in the EFP.

\section{Materials and Methods}

\subsection{Subjects}

Nineteen well-trained eumenorrheic women ( $28.6 \pm 5.9$ years of age, $163.4 \pm 6.1 \mathrm{~cm}$ height, $59.6 \pm$ $5.8 \mathrm{~kg}$ body mass, $14.8 \pm 5.1 \mathrm{~kg}$ fat mass, $42.6 \pm 3.1 \mathrm{~kg}$ fat free mass, $2.6 \pm 0.3 \mathrm{~kg}$ bone mineral content) volunteered to participate in this study. Subjects were recruited by means of diverse advertisements published in social media and regional sport competitions from July 2017 to the end of the study. The applicants were filtered through an initial online questionnaire previously fulfilled with personal information and training experience in order to evaluate the following eligibility criteria: (a) to be between 20-40 years old and (b) to be resistance-trained. All participants self-reported their experience in resistance training-completing resistance workouts of about $60 \pm 20$ minutes, $3 \pm 1$ times per week, during $5.5 \pm 4.9$ years- and they were also involved in other sport activities. The exclusion criteria included: (a) irregular menstrual cycles; (b) use of contraceptives in the six months preceding the study, (c) any existing disease and/or metabolic or hormonal disorder; (d) any musculoskeletal injury in the last six months; (e) any surgical interventions (i.e., ovariectomy) or other medical conditions that would be exacerbated by an eccentric resistance exercise protocol; (f) the regular use of medication or dietary supplements that could affect the results; (g) pregnancies in the year preceding; (h) smoking. In order to calculate sample size, the previous results from the study by Sipavičiene et al., [14] were used, which evaluated the main variable of the study (CK) in two different menstrual cycle phases (EFP and LFP). Taking these data as reference, GPOWER software (GPOWER Version 2, Department of Psychology, Bonn University, Bonn, Germany) was used to perform the analysis, suggesting a sample size of 19 to produce a statistical power of 0.80 with an effect size of 0.75 at a significance level of $p<0.05$. The study consisted of a resistance-based eccentric protocol performed in the three aforementioned menstrual cycle phases (EFP, LFP and MLP) which were adequately randomized and counter-balanced. All procedures complied with the Declaration of Helsinki and the study was approved by the research ethics committee of the Universidad Politécnica de Madrid. A written informed consent was obtained from each participant.

\subsection{Menstrual Cycle Phase Determination}

Eumenorrheic menstrual cycles were defined as regularly occurring menstrual cycles ranging from 24 to 35 days in length [25] in the preceding $\geq 6$ months. Participants confirmed their regular menstruating cycles by providing a retrospective recording of their last six menstrual cycles' length - with the onset of menses considered as the start of the cycle. Following this information, a gynecologist determined the individual's different cycle phases and their average phase length, and prospectively estimated the following cycle. To adequately arrange the trials in the different cycle phases, a home urine-based test (Ellatest, Alicante, Spain) was used to detect urine luteinizing hormone (LH). This is a reliable method of predicting ovulation [26] by detecting the LH surge and the subsequent ovulation naturally occurring within the 14-26 hours [9]. The morning mid-stream urine sample was collected on a daily basis in the three to five days prior to the expected ovulation date, previously estimated by the gynaecologist, until LH surge confirmation. Finally, to correctly verify menstrual cycle phases [22], blood samples were collected for the determination of 17-\$ estradiol, progesterone, FSH and LH in each phase to confirm that the participants were performing the tests in 
the correct menstrual cycle phase. In this regard, ovulation was confidently assured when the minimum conservative limit of $16 \mathrm{nmol} / \mathrm{L}(4.61 \mathrm{ng} / \mathrm{mL})$ of post-ovulatory progesterone was accomplished [11,24].

\subsection{Screening Protocol}

Participants visited the laboratory within days 2 to 5 of their first menstrual bleeding, at the EFP. In this screening session, blood samples were collected to discard the possibility of an existing disorder. Subsequently, participants' body composition was analyzed via a Dual-Energy X-ray Absorptiometry (DEXA) scanner (GE Lunar Prodigy apparatus, GE Healthcare, Madison, WI, USA) using the GE Encore 2002 software ( $\mathrm{v}$ 6.10.029). This screening session concluded with a strength assessment of the lower limbs through a one repetition maximum (1RM) test for the parallel back-squat exercise using a plate-loaded barbell. The 1RM was calculated using an iPhone 6S (Apple Inc., USA) and the Powerlift App [27]. Full range of motion for the lift was always recorded by the same researcher using the Powerlift App [27], with a recording frequency of 240 frames per second. Then, the beginning and the end of the lift was chosen, and the app provided the estimated the 1RM load. According to the manufacturer, these procedures require manual selection by the researcher; thus, two independent observers analyzed the same videos. High interobserver agreement has been shown in previous validation studies (ICC > 0.9) [28].

\subsection{Eccentric-Exercise Sessions}

Participants repeated the same workout on three occasions according to the EFP, LFP and MLP of the menstrual cycle, as these phases present the most pronounced fluctuations in sex hormones [11] (Figure 1). After a 5-min cycle-ergometer warm-up and some mobility and dynamic stretching exercises, the 1RM for the back-squat exercise was calculated in each session by performing a quick test with the Powerlift App based on the full test previously performed in the screening session. The eccentric-based protocol, which was previously used with resistance-trained males, was carried out in the $3 \pm 1$ days, $12 \pm 3$ days and $22 \pm 3$ days of the menstrual cycle, respectively, for the EFP, LFP and MLP (Figure 1).

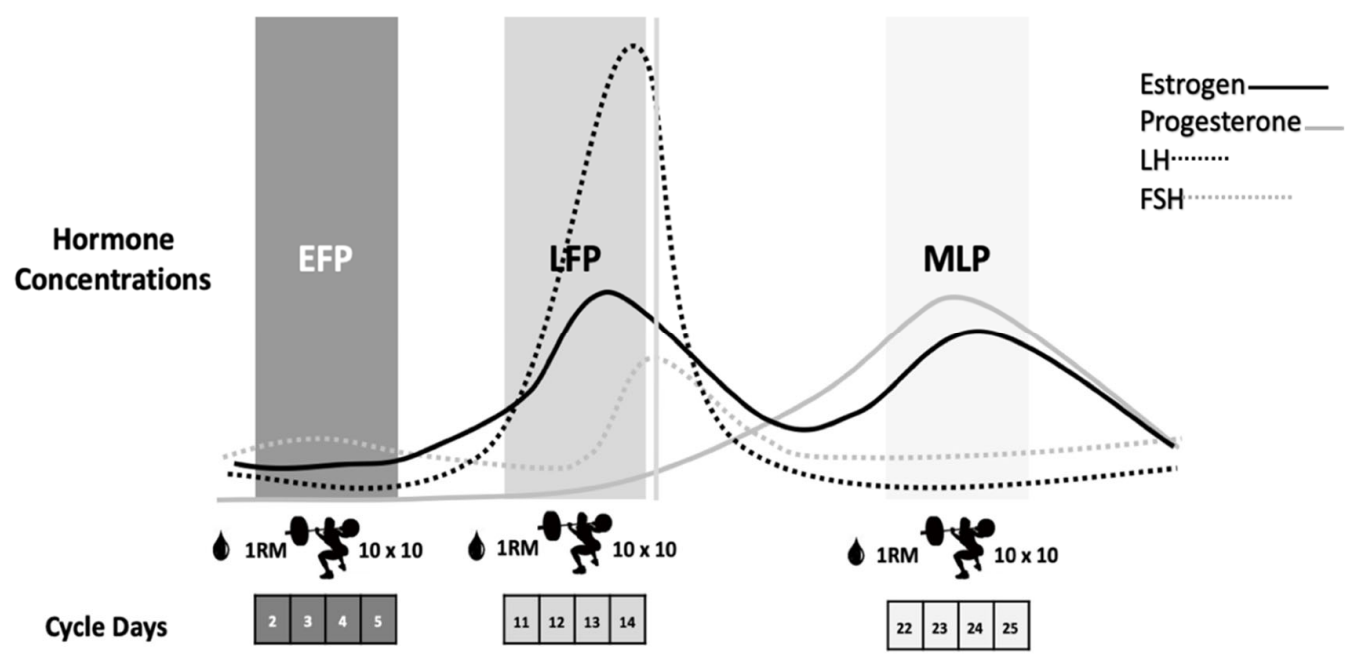

Figure 1. Eccentric-based muscle damaging protocol performed in the EFP, LFP and MLP of the menstrual cycle. EFP: early follicular phase, LFP: late follicular phase, MLP: mid-luteal phase. LH: luteinizing hormone, FSH: follicle stimulating hormone.

It consisted of 10 sets of 10 reps of plate-loaded barbell parallel back squats, at $60 \%$ of their $1 \mathrm{RM}$, with 2 min of rest between sets. Squats were performed at a tempo of 4-s eccentric movement, 1-s pause at the bottom, 1-s concentric movement, and a 1-s pause at the top of the lift to focus on the eccentric phase of the lift for greater muscle damage [29]. The tempo was controlled using an interval timer, with the investigator signaling the changes in the lifting phase and giving verbal encouragement. 
Blood samples were obtained prior to exercise and at $2 \mathrm{~h}, 24 \mathrm{~h}$ and $48 \mathrm{~h}$ post-exercise to analyze muscle damage and inflammation markers: CK, myoglobin, $\mathrm{LDH}, \mathrm{C}$ reactive protein (CRP), TNF- $\alpha, \mathrm{IL}-6$, aspartate aminotransferase (AST) and alanine aminotransferase (ALT).

\subsection{Blood Sampling and Biochemical Analysis}

All blood samples were obtained by venipuncture into a vacutainer containing clot activator. Following inversion and clotting, the blood sample was centrifuged (Biosan LMC-3000 version V.5AD, Riga, Latvia) for ten minutes at $3000 \mathrm{rpm}$ and transferred into Eppendorf tubes and stored frozen at $-80{ }^{\circ} \mathrm{C}$ until further analysis. Follicle stimulating hormone (FSH), LH, progesterone, $17 \beta$ estradiol and IL-6 were measured with a COBAS E411 (Roche Diagnostics, GmbH, Mannheim, Germany), using electrochemiluminescence immunoassay (ECLIA) technology. TNF- $\alpha$ was measured with an IMMULITE 1000 system (Siemens Healthineers AG, Munich, Germany) using chemiluminescent enzymatic immunoassay. Finally, CK, myoglobin, LDH, CRP, ALT and AST were analyzed in a Beckman AU400 Clinical Biochemistry analyzer (Beckman Coulter Inc., Brea, CA, USA). Reactive was calibrated following internal laboratory calibration protocols, and controls were assessed after calibration. Coefficients of variation reported by the laboratory were $4.70 \%$ for FSH, 5.15\% for LH, $4.85 \%$ for 17 - $\beta$ estradiol, $6.35 \%$ for progesterone, $4.10 \%$ for IL-6, $6.50 \%$ for TNF- $\alpha, 6.43 \%$ for CK, $4.17 \%$ for myoglobin, $5.72 \%$ for LDH, 6.40\% for CRP, 7.19\% for AST, and 7.32\% for ALT.

\subsection{Statistical Analysis}

Data are presented as mean \pm SD. Statistical analyses were conducted using the software package SPSS for Windows, version 25.0 (IBM Corp, Armonk, NY). A Saphiro-Wilk test for normality was used. To analyze hormone concentrations and 1RM in each of the three phases, a one-way ANOVA was performed. To explore our objective, a mixed linear model was performed to analyze the repeated measures, setting phase and time as the fixed effects and subjects as the random effect. Where appropriate, the Bonferroni post hoc test was applied to examine pairwise comparisons for each significant factor. Finally, estimated magnitudes of difference in means and their 95\% confidence limits were calculated and presented in standardized units, and were evaluated qualitatively with the following scale: trivial, 0-0.2; small, 0.2-0.6; moderate, 0.6-1.2; large, 1.2-2.0; and very large, >2.0 [30]. Significance level was set at $p<0.05$.

\section{Results}

\subsection{Sex Hormones and Strength Assessment}

Results for the hormonal analysis confirmed that the trials were performed in the correct menstrual cycle phases. A significant effect of menstrual cycle phase was observed for $17 \beta$-estradiol (38.2 \pm 32.1 , $185.1 \pm 173.9$ and $156.1 \pm 91.5 \mathrm{pg} / \mathrm{mL})$, progesterone $(0.3 \pm 0.1,0.4 \pm 0.7$ and $10.1 \pm 3.9 \mathrm{ng} / \mathrm{mL}), \mathrm{FSH}$ $(7.1 \pm 2.5,6.1 \pm 2.9$ and $3.1 \pm 1.2 \mathrm{mUI} / \mathrm{mL})(p<0.001$ for all variables $)$, and $\mathrm{LH}(6.0 \pm 1.9,11.9 \pm 10.3$ and $4.4 \pm 2.4 \mathrm{mUI} / \mathrm{mL} ; p=0.008)$, respectively for the EFP, LFP and MLP. Results for the 1RM were $75.9 \pm 16.8 \mathrm{~kg}, 75.5 \pm 17.4 \mathrm{~kg}$ and $76.0 \pm 16.8 \mathrm{~kg}$ for EFP, LFP and MLP, respectively, and reported no differences between phases $(p>0.05)$.

\subsection{Muscle Damage}

Muscle damage markers results and pairwise comparisons are shown in Table 1. Results showed no effect of menstrual cycle phase in CK $\left(133.8 \pm 12.1,146.7 \pm 12.1\right.$ and $\left.138.1 \pm 12.1 \mathrm{U} \cdot \mathrm{L}^{-1}\right)$ myoglobin $\left(73.2 \pm 2.9,79.6 \pm 3.7\right.$ and $\left.74.1 \pm 2.8 \mu \cdot \mathrm{L}^{-1}\right), \mathrm{LDH}\left(170.9 \pm 3.1,169.3 \pm 4.6\right.$ and $\left.170.5 \pm 3.1 \mathrm{U} \cdot \mathrm{L}^{-1}\right)$ and AST $\left(22.4 \pm 1.0,23.2 \pm 1.1\right.$ and $\left.23.3 \pm 0.9 \mathrm{U} \cdot \mathrm{L}^{-1}\right)$ respectively for the EFP, LFP and MLP $(p>0.05$ for all variables). The analysis of ES revealed low results when comparing menstrual cycle phases (Figure 2). In contrast, a trend for menstrual cycle phase was observed for ALT, $(16.3 \pm 1.0,17.3 \pm 0.9$ and $\left.23.7 \pm 0.9 \mathrm{Ui} / \mathrm{L} ; \mathrm{F}_{2,49}=3.067 ; p=0.056\right)$ indicating that in the LFP, ALT values were almost higher 
than in MLP ( $p=0.068)$, but ES was not meaningful (Figure 2). No significant interaction between time and menstrual cycle phase was observed for all these markers and the ES was negligible (data not shown).

Table 1. Blood markers of muscle damage and inflammation throughout the menstrual cycle following an eccentric-based resistance exercise. Data expressed as mean (SD).

\begin{tabular}{|c|c|c|c|c|c|c|c|c|}
\hline & \multicolumn{2}{|c|}{ EFP } & \multicolumn{2}{|c|}{ LFP } & \multicolumn{2}{|c|}{ MLP } & \multicolumn{2}{|c|}{ TOTAL } \\
\hline \multicolumn{9}{|c|}{ CK $\left(U \cdot L^{-1}\right)$} \\
\hline Baseline & 108.6 & $(48.0)$ & 105.7 & $(33.1)$ & 100.7 & (29.9) & 105.0 & (37.3) \\
\hline $2 \mathrm{~h}$ & 151.6 & $(70.0)$ & 155.1 & $(44.9)$ & 150.6 & $(43.8)$ & $152.4^{*}$ & (53.3) \\
\hline $24 \mathrm{~h}$ & 154.1 & (69.3) & 195.5 & (95.3) & 172.1 & (85.8) & $173.9^{\mathrm{a}}$ & (84.4) \\
\hline $48 \mathrm{~h}$ & 117.3 & $(40.1)$ & 130.6 & $(47.7)$ & 128.8 & $(49.5)$ & $125.6^{a, c}$ & $(45.5)$ \\
\hline Total & 132.9 & $(60.7)$ & 146.7 & $(67.7)$ & 138.1 & $(61.1)$ & & \\
\hline \multicolumn{9}{|c|}{ Myoglobin $(\mu \mathrm{g} \cdot \mathrm{L})$} \\
\hline Baseline & 62.8 & $(8.2)$ & 60.4 & $(7.2)$ & 60.1 & $(10.6)$ & 61.1 & $(8.9)$ \\
\hline $2 \mathrm{~h}$ & 105.5 & $(43.9)$ & 129.1 & $(56.3)$ & 107.9 & $(41.2)$ & $115.0^{* *}$ & $(48.7)$ \\
\hline $24 \mathrm{~h}$ & 64.5 & $(9.6)$ & 64.9 & $(7.9)$ & 66.1 & $(10.7)$ & 65.2 & $(9.6)$ \\
\hline $48 \mathrm{~h}$ & 59.8 & $(7.4)$ & 63.8 & $(9.0)$ & 62.2 & $(8.1)$ & 61.9 & (8.5) \\
\hline Total & 73.2 & $(30.0)$ & 79.6 & $(41.0)$ & 74.1 & (30.1) & & \\
\hline \multicolumn{9}{|c|}{ LDH $\left(U \cdot L^{-1}\right)$} \\
\hline Baseline & 166.5 & (14.7) & 164.3 & $(22.6)$ & 163.9 & (13.4) & 164.9 & (17.4) \\
\hline $2 \mathrm{~h}$ & 187.1 & (20.3) & 181.5 & (26.8) & 184.4 & (16.3) & $184.3^{* *}$ & (21.3) \\
\hline $24 \mathrm{~h}$ & 166.2 & (15.9) & 170.2 & (23.9) & 165.7 & (17.4) & 167.4 & (19.1) \\
\hline $48 \mathrm{~h}$ & 163.8 & (16.5) & 161.4 & (16.2) & 167.9 & (16.9) & 164.4 & (16.5) \\
\hline Total & 170.9 & (19.1) & 169.3 & (23.6) & 170.7 & (18.9) & & \\
\hline \multicolumn{9}{|c|}{ IL-6 (pg/mL) } \\
\hline Baseline & 1.7 & $(0.7)$ & 1.7 & $(0.5)$ & 1.6 & $(0.3)$ & 1.7 & $(0.6)$ \\
\hline $2 \mathrm{~h}$ & 1.8 & $(0.7)$ & 1.7 & $(0.6)$ & 2.0 & (1.3) & 1.9 & (0.9) \\
\hline $24 \mathrm{~h}$ & 1.6 & $(0.2)$ & 1.7 & $(0.7)$ & 1.5 & $(0.0)$ & $1.6^{\mathrm{b}}$ & $(0.4)$ \\
\hline $48 \mathrm{~h}$ & 1.6 & $(0.5)$ & 1.9 & $(0.7)$ & 1.5 & $(0.1)$ & 1.7 & $(0.4)$ \\
\hline Total & 1.7 & $(0.5)$ & 1.7 & $(0.6)$ & 1.7 & $(0.7)$ & & \\
\hline \multicolumn{9}{|c|}{ TNF- $\alpha(\mathrm{pg} / \mathrm{mL})$} \\
\hline Baseline & 4.9 & (1.1) & 5.3 & $(1.9)$ & 4.9 & (2.0) & 5.0 & (1.7) \\
\hline $2 \mathrm{~h}$ & 5.2 & (1.4) & 5.3 & (1.6) & 4.9 & (1.4) & 5.2 & (1.5) \\
\hline $24 \mathrm{~h}$ & 4.8 & (1.4) & 4.8 & (1.0) & 4.6 & (0.9) & $4.7^{\# \#}$ & (1.1) \\
\hline $48 \mathrm{~h}$ & 4.5 & $(0.9)$ & 4.6 & (0.9) & 4.7 & (1.2) & 4.6 & (1.0) \\
\hline Total & 4.8 & (1.2) & 5.0 & (1.4) & 4.8 & (1.4) & & \\
\hline \multicolumn{9}{|c|}{ CRP (mg/L) } \\
\hline Baseline & 0.6 & $(0.3)$ & 0.6 & $(0.3)$ & 0.5 & $(0.3)$ & 0.5 & $(0.3)$ \\
\hline $2 \mathrm{~h}$ & 0.5 & (0.3) & 0.5 & $(0.3)$ & 0.5 & $(0.3)$ & 0.5 & $(0.3)$ \\
\hline $24 \mathrm{~h}$ & 0.6 & $(0.4)$ & 0.5 & (0.3) & 0.5 & (0.3) & $0.5^{\# \#}$ & (0.3) \\
\hline $48 \mathrm{~h}$ & 0.5 & $(0.3)$ & 0.5 & (0.3) & 0.5 & $(0.3)$ & $0.5^{b, \#}$ & $(0.3)$ \\
\hline Total & 0.5 & $(0.4)$ & 0.5 & (0.3) & 0.5 & (0.3) & & \\
\hline \multicolumn{9}{|c|}{ AST (Ui/L) } \\
\hline Baseline & 21.2 & (4.4) & 21.8 & $(5.0)$ & 22.6 & (5.1) & 21.8 & $(4.8)$ \\
\hline $2 \mathrm{~h}$ & 22.8 & $(4.2)$ & 23.4 & (4.7) & 23.8 & $(4.2)$ & $23.4^{\mathrm{a}}$ & (4.3) \\
\hline $24 \mathrm{~h}$ & 23.1 & (4.7) & 24.3 & (5.1) & 23.8 & (4.0) & $23.7^{\mathrm{a}}$ & (4.6) \\
\hline $48 \mathrm{~h}$ & 22.4 & (5.2) & 23.3 & (5.4) & 22.8 & (4.6) & 22.8 & (5.0) \\
\hline Total & 22.4 & (4.6) & 23.2 & (5.0) & 23.3 & (4.5) & & \\
\hline \multicolumn{9}{|c|}{ ALT (Ui/L) } \\
\hline Baseline & 16.0 & $(4.5)$ & 16.8 & $(5.5)$ & 14.7 & $(4.9)$ & 15.8 & (5.1) \\
\hline $2 \mathrm{~h}$ & 16.3 & (4.4) & 17.3 & (5.4) & 15.6 & (4.1) & 16.4 & (4.7) \\
\hline $24 \mathrm{~h}$ & 16.4 & (4.5) & 17.4 & (5.3) & 15.8 & (3.9) & 16.6 & (4.6) \\
\hline $48 \mathrm{~h}$ & 16.4 & (5.1) & 17.5 & (5.9) & 15.9 & (4.4) & 16.6 & (5.1) \\
\hline Total & 16.3 & (4.5) & 17.3 & (5.4) & $15.5 \S$ & (4.4) & & \\
\hline
\end{tabular}

EFP: early follicular phase, LFP: late follicular phase, MLP: mid-luteal phase, CK: creatine kinase. LDH: lactate dehydrogenase; IL-6: interleukin-6; TNF- $\alpha$ : tumor necrosis factor; CRP: $C$ reactive protein; AST: aspartate aminotransferase; ALT: alanine aminotransferase; ${ }^{*}$ Different from the rest of time-points $(p<0.05) ;{ }^{* *}$ Different from the rest of time points $(p<0.001){ }^{a}$ Different from pre-trial $(p<0.05) ;{ }^{b}$ Different from $2 \mathrm{~h}$ post-exercise $(p<0.05)$. ${ }^{c}$ Different from $24 \mathrm{~h}$ post-exercise; $(p<0.05)$. \# Trend is different from pre-trial $(p=0.059)$; ${ }^{\#}$ Trend is different from $2 \mathrm{~h}(p=0.064$ and $p=0.084$, respectively, for TNF- $\alpha$ and CRP); $\S$ Trend is different from LFP $(p=0.068)$. 


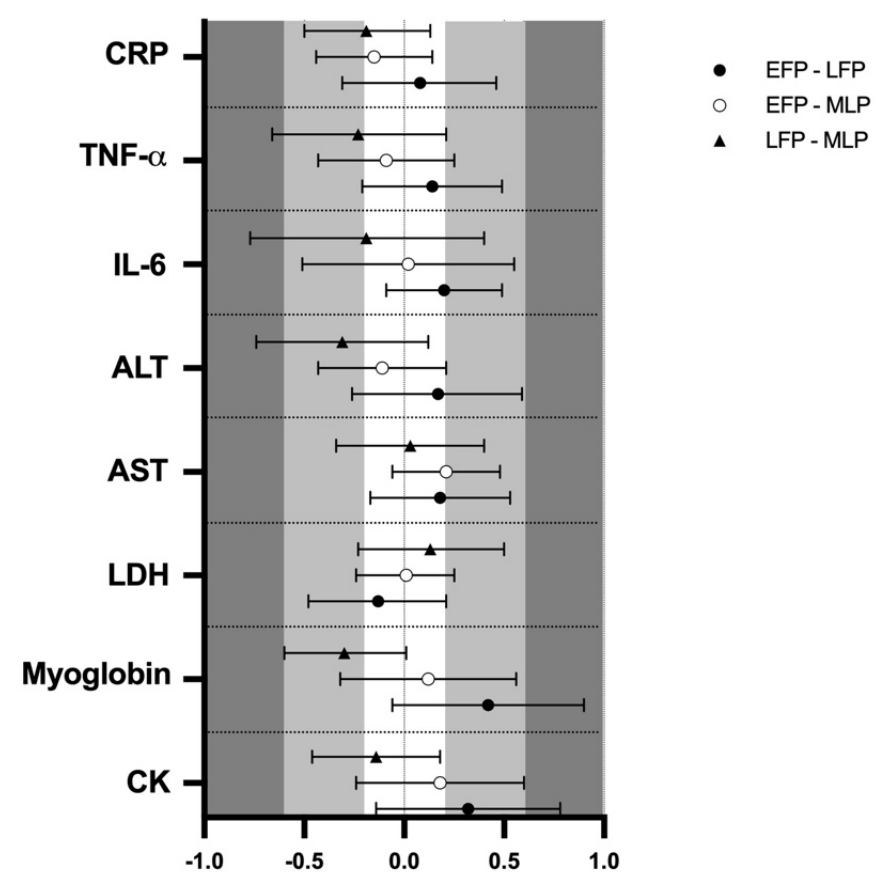

Figure 2. Standardized mean differences (ES) and confidence intervals in muscle damage and inflammation markers among menstrual cycle phases. EFP: early follicular phase, LFP: late follicular phase, MLP: mid-luteal phase. CK: creatine kinase, LDH: lactate dehydrogenase, AST: aspartate aminotransferase, ALT: alanine aminotransferase, IL-6: interleukin-6, TNF- $\alpha$ : tumor necrosis factor $\alpha$, CRP: c reactive protein.

Finally, a significant effect of time ( $p<0.001$ for all variables) was observed in CK $(105.0 \pm 6.2$, $152.4 \pm 10.6,173.8 \pm 16.9$ and $\left.125.6 \pm 9.0 \mathrm{U} \cdot \mathrm{L}^{-1} ; \mathrm{F}_{3,161}=37.543\right)$, myoglobin $(61.1 \pm 1.5,114.2 \pm 9.0$, $65.2 \pm 1.7$ and $\left.61.9 \pm 1.4 \mu \cdot \mathrm{L}^{-1} ; \mathrm{F}_{3,150}=63.747\right), \mathrm{LDH}(164.9 \pm 3.4,184.3 \pm 4.1,167.3 \pm 3.6$ and $\left.164.4 \pm 3.3 \mathrm{U} \cdot \mathrm{L}^{-1} ; \mathrm{F}_{3,154}=33.883\right)$ and AST $(21.8 \pm 0.9,23.4 \pm 1.2,23.7 \pm 0.9$ and $22.8 \pm 1.1 \mathrm{Ui} / \mathrm{L}$; $\mathrm{F}_{3,162}=6.514$ ), respectively at pre-trial, $0 \mathrm{~h}, 24 \mathrm{~h}$ and $48 \mathrm{~h}$, supported by the ES (Figure 3A,B). No effect of time was observed for ALT $(21.8 \pm 1.0,23.4 \pm 0.9,23.7 \pm 0.9$ and $22.8 \pm 1.1 \mathrm{Ui} / \mathrm{L} ; p<0.05)$.
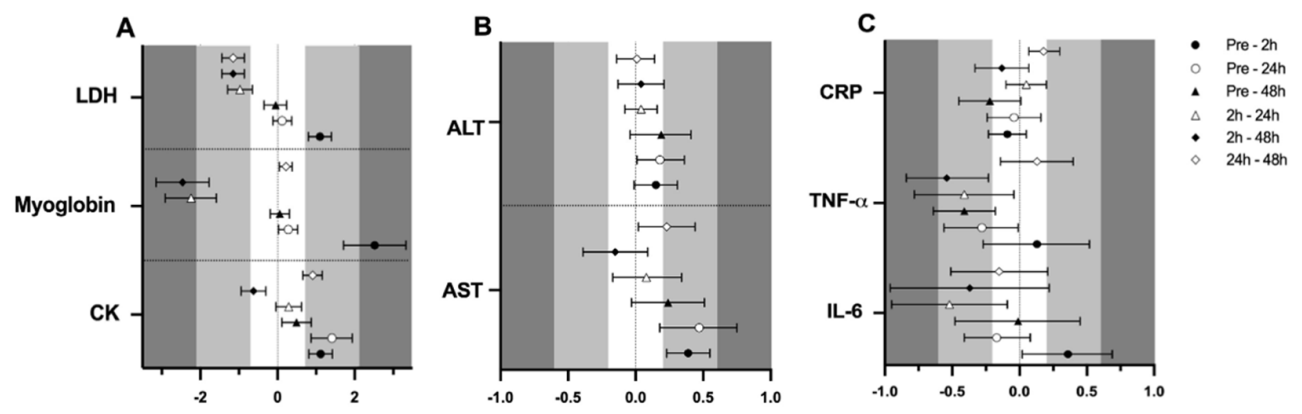

Figure 3. Standardized mean differences (ES) and confidence intervals in muscle damage and inflammation markers among time-point measurements. (A)CK, myoglobin and LDH; (B)AST and ALT; (C)IL-6, TNF- $\alpha$ and CRP; EFP: early follicular phase, LFP: late follicular phase, MLP: mid-luteal phase. CK: creatine kinase, LDH: lactate dehydrogenase, AST: aspartate aminotransferase, ALT: alanine aminotransferase, IL-6: interleukin-6, TNF- $\alpha$ : tumor necrosis factor $\alpha$, CRP: c reactive protein.

\subsection{Inflammation}

Regarding inflammation markers, results are also presented in Table 1 . Results showed no effect of menstrual cycle phase in IL-6 $(1.7 \pm 0.4,1.8 \pm 0.5$ and $1.7 \pm 0.4 \mathrm{pg} / \mathrm{mL}), \mathrm{TNF}-\alpha(4.8 \pm 0.9,5.0 \pm 1.2$ and $4.8 \pm 1.2 \mathrm{pg} / \mathrm{mL})$ or CRP $(0.52 \pm 0.07,0.54 \pm 0.06$ and $0.47 \pm 0.06 \mathrm{mg} / \mathrm{L})$, respectively for the EFP, LFP 
and MLP ( $p>0.05$ for all variables); while the ES was low for menstrual cycle phase-effects (Figure 2). An interaction between time and menstrual cycle phase $\left(\mathrm{F}_{6,150}=2.192 ; p=0.047\right)$ was observed for IL6. Accordingly, a moderate ES indicated that only in the MLP were $2 \mathrm{~h}$ post-exercise values higher than pre-trial [0.68 (0.53)], $24 \mathrm{~h}[0.84(0.74)]$ and $48 \mathrm{~h} \mathrm{[0.75(0.75)].} \mathrm{This} \mathrm{effect} \mathrm{was} \mathrm{not} \mathrm{observed} \mathrm{in} \mathrm{the} \mathrm{EFP}$ and LFP. No interaction or meaningful ES was observed between time and menstrual cycle phase for CRP and TNF- $\alpha$ (data not shown). A significant effect of time for IL-6 $(1.7 \pm 0.4,1.9 \pm 0.7,1.6 \pm 0.3$ and $\left.1.7 \pm 0.3 \mathrm{pg} / \mathrm{mL} ; \mathrm{F}_{3,154}=2.955 ; p=0.034\right)$, TNF- $\alpha(5.0 \pm 1.3,5.2 \pm 1.1,4.7 \pm 0.9$ and $4.6 \pm 0.9 \mathrm{pg} / \mathrm{mL}$; $\left.\mathrm{F}_{3,156}=3.958 ; p=0.009\right)$ and CRP $\left(0.53 \pm 0.06,0.51 \pm 0.06,0.54 \pm 0.07\right.$ and $0.47 \pm 0.06 \mathrm{mg} / \mathrm{L} ; \mathrm{F}_{3,159}=$ 2.759; $p=0.044$ ) was obtained for pre-trial, $0 \mathrm{~h}, 24 \mathrm{~h}$ and $48 \mathrm{~h}$, respectively. A moderate ES indicated that TNF- $\alpha$ concentrations at $2 \mathrm{~h}$ seemed to be higher in comparison to $48 \mathrm{~h}$ (Figure 3C).

\section{Discussion}

The major finding of this study is that the hormonal environment throughout the different menstrual cycle phases did not affect blood markers of muscle damage and inflammation. The only variable showing a possible exercise-induced inflammatory response was IL-6 in the MLP; although this finding should be taken with caution. This workout based on eccentric squats elicited muscle damage, as could be observed from post-exercise concentrations of CK, myoglobin and LDH. In addition, in a previous study conducted by our laboratory, high post-exercise muscle soreness was also observed in the same sample following an identical exercise protocol (unpublished data). However, this exercise-induced damaging exercise was not strenuous enough to trigger inflammation. This lack of menstrual cycle phase differences in blood markers of muscle damage is potentially due to the fact that our participants were well-trained in resistance training [31]. This is in agreement with a previous study which also obtained no differences between the EFP and LFP in serum CK following a drop jump protocol [16]. They indicated that previous exposures to eccentric exercise can influence the $\mathrm{CK}$ response, suggesting that $\mathrm{CK}$ concentration is an unreliable marker of muscle damage in training studies where the same muscle groups are exercised in a subsequent session [32]. Another possible explanation could be the high variability in the response to a muscle-damaging exercise. This is associated with the presence of some polymorphisms present in genes encoding for the myofibrillar proteins $\alpha$-actinin 3 and myosin light chain kinase, which influence CK and myoglobin responses [28]. Altogether, this might indicate that higher estrogen concentrations during LFP and MLP may not provide extra protection against muscle damage in comparison to the EFP as the literature suggests $[4,5,11,15]$.

Regarding the effect of menstrual cycle phase on inflammation response, IL-6 values seem to be higher $2 \mathrm{~h}$ post-exercise than at the other time-points, hence indicating a likely inflammatory response to exercise, only observed in the MLP. In fact, from some previous studies indicating that estrogen has a role in the regulation of immunocompetence [33-36], most of them reported an upregulated pro-inflammatory response to exercise in the luteal phase in comparison to the mid/late follicular phases $[35,36]$. However, it may possible that these gene expression changes in response to exercise, may represent a swift return to baseline from a highly anti-inflammatory state at rest, as opposed to presenting a truly pro-inflammatory response. Another possible reason may be the presence of progesterone during the MLP which may interact with certain estrogen-protective responses, resulting in an increase in inflammation. This interaction between estrogen and progesterone has been observed in other endocrine responses [37]. Interestingly, the increase in IL-6 at $2 \mathrm{~h}$ in the MLP was not preceded by an increase in TNF- $\alpha$. Therefore, such an increase in IL- 6 might be not attributed to the inflammatory response but to the endocrine role of muscle, which enhances IL-6 concentrations after glycogen depletion during exercise [15]. However, on the basis of IL-6 as a myokine, the highest IL-6 concentrations have particularly been observed at the cessation or 30-min post exercise [38,39]. In addition, it should be taken into consideration that TNF- $\alpha$ rarely increases in response to exercise, except for highly strenuous prolonged efforts such as marathons [40]. Thus, the potential role of IL-6 as a myokine could be doubtful, with the data indicating a more likely inflammatory role. 
Another factor possibly involved in IL-6 changes may be the modality of exercise. A previous study obtained higher values of IL-6 in the mid-follicular phase in comparison to the MLP after a continuous running protocol [10], while a different study obtained no differences between the EFP and the MLP following a running protocol [15]. As such, more research is needed to clarify whether different exercise modalities could facilitate different increases in IL-6 in relation to menstrual cycle phase. In line with that, our lacking time-differences in IL-6 concentration between pre-trial and $2 \mathrm{~h}$ could be also due to the exercise modality. In the present exercise protocol, stimulus duration as well as the muscle mass involved in the exercise may be insufficient to increase IL-6 from pre-trial levels [15]. This has been demonstrated following aerobic protocols [40] which are generally long-lasting and involve larger muscle groups. In fact, our findings revealed that IL- 6 and TNF- $\alpha$ concentrations decreased after $24 \mathrm{~h}$ in comparison to $2 \mathrm{~h}$ independent of the phase studied. This, however, is unlikely to be associated with exercise due to the aforementioned absence of differences between pre-trial and $2 \mathrm{~h}$ measurements. Interestingly, despite the lacking inflammatory effect reported, a remarkably moderate effect might suggest reductions in TNF- $\alpha$ at $48 \mathrm{~h}$ in comparison to $2 \mathrm{~h}$ which could be in agreement with some of the anti-inflammatory effects of exercise, as indicated in the literature [40].

Lastly, menstrual cycle phases do not seem to affect AST and ALT; however, it is challenging to discuss this finding due to the lack of literature evaluating these enzymes during the various menstrual cycle phases. Interestingly, our ALT values appear to be higher in the LFP for all time-points. Although ALT is mainly located in the liver, alanine synthesis also occurs in the muscle [41]. Then, according to these results, participants' higher estrogen concentrations during the LFP may predispose participants' muscle tissue to release this enzyme as a consequence of muscle injury due to an exercise overload. However, our values are within normative values and do not appear to be indicative of muscle injury. In fact, ALT increases have been reported following extreme physical exertion such as Thai boxing or ultramarathons [41], so it is very unlikely that our squat-based protocol elicited such a response. It has also been suggested that pro-inflammatory cytokines may exert a catabolic effect on muscle, which would facilitate the increase of ALT [42]. Thus, the higher ALT concentration in the LFP would agree with some results that suggest the lack of estrogen's protective effect, but further investigation is warranted. On the contrary, the AST response was not affected by menstrual cycle phases but increased in post-exercise measurements, which is in accordance with previous female studies $[43,44]$.

To the best of our knowledge, this is the first study to investigate muscle damage and inflammation responses induced by an eccentric exercise focused on squats, in the same group of participants, and considering the menstrual cycle phases with the most pronounced changes in hormonal concentrations. As far as we are concerned, the current study presents an adequate methodology to verify menstrual cycle phases: hormonal analysis, calendar counting and LH confirmation via urine-based tests. Intriguingly, despite being a robust methodology, and a gynecologist confirmed the regularity of participants' menstrual cycles, individual variation in muscle damage and inflammation markers was typically highly variable. This may be a confounding factor to observe differences between menstrual cycle phases and the results should be taken cautiously. Additionally, the choice of the exercise protocol may have also been troublesome to elicit noteworthy differences between menstrual cycle phases. Despite this squat-based workout -that could be incorporated into regular female athletes- was previously performed to elicit muscle damage [29], it barely triggered muscle damage, and hence, inflammation response in our sample. The training status of our well-trained participants may have affected exercise-induced muscle damage development. Finally, as a result of this design, previous studies in the literature are not entirely comparable to ours and it could also be considered as a limitation. However, despite the aforementioned drawbacks the findings provided by this study could contribute to elucidate the lacking influence of sex hormones on muscle damage and inflammation, following a squat-based workout potentially usable by coaches on a regular basis. 


\section{Conclusions}

In conclusion, no differences between menstrual cycle phases are observed in blood markers of muscle damage and inflammation following a resistance training focused on eccentric squats. The only phase to report a possible inflammation-related pattern in terms of IL-6 was the MLP. However, the lack of differences in estrogen between the LFP and the MLP make it difficult to relate this finding to the lack of estrogen's protective effect. Further research should focus on homogenizing phases measured and verification in order to properly clarify the influence of hormonal status on blood markers of muscle damage and inflammation.

Author Contributions: Conceptualization, A.B.P. and R.C.; methodology, A.B.P., R.C., L.B.-M. and N.R.-P.; software, N.R.-P.; validation, L.B.-M.; formal analysis, N.R.-P. and A.B.P.; investigation N.R.-P., V.M.A.-M., B.R., Á.E.D.; resources, A.B.P. and F.J.C.; data curation, N.R.-P., V.M.A.-M., B.R. and L.B.-M.; writing-original draft preparation, N.R.-P.; writing—review and editing, N.R.-P., L.B.-M., V.M.A.-M., B.R., F.J.C., Á.E.D., R.C., A.B.P.; visualization N.R.-P.; supervision A.B.P.; project administration, A.B.P.; funding acquisition A.B.P. All authors have read and agreed to the published version of the manuscript.

Funding: The IronFEMME Study takes place with the financial support of the Ministerio de Economía y Competitividad, Convocatoria de ayudas I + D 2016, Plan Estatal de Investigación Científica y Técnica y de Innovación 2013-2016 (Contract DEP2016-75387-P). N.R-P and V.M.A-M are both supported by a grant provided by Universidad Politécnica de Madrid.

Acknowledgments: Authors want to acknowledge Nuno Koch Esteves for the language assistance.

Conflicts of Interest: The authors declare no conflict of interest. The funders had no role in the design of the study; in the collection, analyses, or interpretation of data; in the writing of the manuscript, or in the decision to publish the results.

\section{References}

1. Peake, J.M.; Neubauer, O.; Della Gatta, P.A.; Nosaka, K. Muscle damage and inflammation during recovery from exercise. J. Appl. Phys. 2017, 122, 559-570. [CrossRef]

2. Koch, A.J.; Pereira, R.; Machado, M. The creatine kinase response to resistance exercise. J. Musculoskelet. Neuronal Interact. 2014, 14, 68-77. [PubMed]

3. Brancaccio, P.; Maffulli, N.; Limongelli, F.M. Creatine kinase monitoring in sport medicine. Br. Med. Bull. 2007, 81-82, 209-230. [CrossRef] [PubMed]

4. Kendall, B.; Eston, R. Exercise-induced muscle damage and the potential protective role of estrogen. Sports Med. 2002, 32, 103-123. [CrossRef]

5. Enns, D.L.; Tiidus, P.M. The influence of estrogen on skeletal muscle. Sports Med. 2010, 40, 41-58. [CrossRef]

6. Rankin, P.; Lawlor, M.J.; Hills, F.A.; Bell, P.G.; Stevenson, E.J.; Cockburn, E. The effect of milk on recovery from repeat-sprint cycling in female team-sport athletes. Appl. Physiol. Nutr. Metab. 2018, 43, 113-122. [CrossRef]

7. Greising, S.M.; Baltgalvis, K.A.; Lowe, D.A.; Warren, G.L. Hormone therapy and skeletal muscle strength: A meta-analysis. J. Gerontol. Ser. A Biol. Sci. Med. Sci. 2009, 64, 1071-1081. [CrossRef]

8. Dieli-Conwright, C.M.; Spektor, T.M.; Rice, J.C.; Schroeder, E.T. Hormone therapy attenuates exercise-induced skeletal muscle damage in postmenopausal women. J. Appl. Physiol. 2009, 107, 853-858. [CrossRef]

9. Bruinvels, G.; Burden, R.J.; McGregor, A.J.; Ackerman, K.E.; Dooley, M.; Richards, T.; Pedlar, C. Sport, exercise and the menstrual cycle: Where is the research? Br. J. Sports Med. 2017, 51, 487-488. [CrossRef]

10. Hackney, A.C.; Kallman, A.L.; Ağgön, E. Female sex hormones and the recovery from exercise: Menstrual cycle phase affects responses. Biomed. Hum. Kinet. 2019, 11, 87-89. [CrossRef]

11. Janse de Jonge, X.A.K. Effects of the menstrual cycle on exercise performance. Sports Med. 2003, 33, 833-851. [CrossRef]

12. Anderson, L.J.; Baker, L.L.; Schroeder, E.T. Blunted myoglobin and quadriceps soreness after electrical stimulation during the luteal phase or oral contraception. Res. Q. Exerc. Sport 2017, 88, 193-202. [CrossRef]

13. Oosthuyse, T.; Bosch, A. The effect of gender and menstrual phase on serum creatine kinase activity and muscle soreness following downhill Running. Antioxidants 2017, 6, 16. [CrossRef] 
14. Williams, T.; Walz, E.; Lane, A.R.; Pebole, M.; Hackney, A.C. The effect of estrogen on muscle damage biomarkers following prolonged aerobic exercise in eumenorrheic women. Biol. Sport 2015, 32, 193-198. [CrossRef]

15. Chaffin, M.E.; Davis, J.E.; Berg, K.E.; French, J.A.; Meendering, J.R.; Llewellyn, T.L. Interleukin-6 and delayed onset muscle soreness do not vary during the menstrual cycle. Res. Q. Exerc. Sport 2011, 82, 693-701. [CrossRef]

16. Sipavičienè, S.; Daniusevičiutè, L.; Klizienè, I.; Kamandulis, S.; Skurvydas, A. Effects of estrogen fluctuation during the menstrual cycle on the response to stretch-shortening exercise in females. BioMed Res. Int. 2013, 2013, 1-6. [CrossRef]

17. Hicks, K.M.; Onambele-Pearson, G.L.; Winwood, K.; Morse, C.I. Muscle-tendon unit properties during eccentric exercise correlate with the creatine kinase response. Front. Physiol. 2017, 8, 657. [CrossRef]

18. Minahan, C.; Joyce, S.; Bulmer, A.C.; Cronin, N.; Sabapathy, S. The influence of estradiol on muscle damage and leg strength after intense eccentric exercise. Eur. J. Appl. Physiol. 2015, 115, 1493-1500. [CrossRef]

19. Hicks, K.M.; Onambélé, G.L.; Winwood, K.; Morse, C.I. Muscle Damage following Maximal Eccentric Knee Extensions in Males and Females. PLoS ONE 2016, 11, e0150848. [CrossRef]

20. Joyce, S.; Sabapathy, S.; Bulmer, A.C.; Minahan, C. The effect of prior eccentric exercise on heavy-intensity cycling: The role of gender and oral contraceptives. Eur. J. Appl. Physiol. 2014, 114, 995-1003. [CrossRef]

21. Brown, M.A.; Howatson, G.; Keane, K.; Stevenson, E.J. Undefined, Exercise-induced muscle damage following dance and sprint specific exercise in females. J. Sports Med. Phys. Fit. 2016, 56, 1376-1383.

22. Nikolaidis, M.G.; Paschalis, V.; Giakas, G.; Fatouros, I.G.; Sakellariou, G.K.; Theodorou, A.A.; Koutedakis, Y.; Jamurtas, A.Z. Favorable and prolonged changes in blood lipid profile after muscle-damaging exercise. Med. Sci. Sports Exerc. 2008, 40, 1483-1489. [CrossRef] [PubMed]

23. Stupka, N.; Lowther, S.; Chorneyko, K.; Bourgeois, J.M.; Hogben, C.; Tarnopolsky, M.A. Gender differences in muscle inflammation after eccentric exercise. J. Appl. Physiol. 2000, 89, 2325-2332. [CrossRef] [PubMed]

24. Janse de Jonge, X.A.K.; Thompson, B.; Han, A. Methodological Recommendations for Menstrual Cycle Research in Sports and Exercise. Med. Sci. Sports Exerc. 2019, 51, 2610-2617. [CrossRef]

25. Lebrun, C.M.; McKenzie, D.C.; Prior, J.C.; Taunton, J.E. Effects of menstrual cycle phase on athletic performance. Med. Sci. Sports Exerc. 1995, 27, 437-444. [CrossRef]

26. Miller, P.B.; Soules, M.R. The usefulness of a urinary LH kit for ovulation prediction during menstrual cycles of normal women. Obstet. Gynecol. 1996, 87, 13-17. [CrossRef]

27. Balsalobre-Fernández, C.; Marchante, D.; Baz-Valle, E.; Alonso-Molero, I.; Jiménez, S.L.; Muñóz-López, M. Analysis of wearable and smartphone-based technologies for the measurement of barbell velocity in different resistance training exercises. Front. Physiol. 2017, 8, 649. [CrossRef]

28. Balsalobre-Fernández, C.; Marchante, D.; Muñoz-López, M.; Jiménez, S.L. Validity and reliability of a novel iPhone app for the measurement of barbell velocity and 1RM on the bench-press exercise. J. Sports Sci. 2018, 36, 64-70. [CrossRef]

29. Macdonald, G.Z.; Button, D.C.; Drinkwater, E.J.; Behm, D.G. Foam rolling as a recovery tool after an intense bout of physical activity. Med. Sci. Sports Exerc. 2014, 46, 131-142. [CrossRef]

30. Hopkins, W.G.; Marshall, S.W.; Batterham, A.M.; Hanin, J. Progressive statistics for studies in sports medicine and exercise science. Med. Sci. Sports Exerc. 2009, 41, 3-13. [CrossRef]

31. Hyldahl, R.D.; Chen, T.C.; Nosaka, K. Mechanisms and Mediators of the Skeletal Muscle Repeated Bout Effect. Exerc. Sport Sci. Rev. 2017, 45, 24-33. [CrossRef] [PubMed]

32. Kamandulis, S.; Skurvydas, A.; Snieckus, A.; Masiulis, N.; Aagaard, P.; Dargeviciute, G.; Brazaitis, M. Monitoring markers of muscle damage during a 3 week periodized drop-jump exercise programme. J. Sports Sci. 2011, 29, 345-353. [CrossRef] [PubMed]

33. Abbasi, A.; de Paula Vieira, R.; Bischof, F.; Walter, M.; Movassaghi, M.; Berchtold, N.C.; Niess, A.M.; Cotman, C.W.; Northoff, H. Sex-specific variation in signaling pathways and gene expression patterns in human leukocytes in response to endotoxin and exercise. J. Neuroinflamm. 2016, 13, 289. [CrossRef] [PubMed]

34. Fish, E.N. The X-files in immunity: Sex-based differences predispose immune responses. Nat. Rev. Immunol. 2008, 8, 737-744. [CrossRef]

35. Northoff, H.; Symons, S.; Zieker, D.; Schaible, E.V.; Schaefer, K.; Thoma, S.; Loeffler, M.; Abbasi, A.; Simon, P.; Niess, A.M.; et al. Gender- and menstrual phase dependent regulation of inflammatory gene expression in response to aerobic exercise. Exerc. Immunol. Rev. 2008, 14, 86-103. 
36. Timmons, B.W.; Hamadeh, M.J.; Devries, M.C.; Tarnopolsky, M.A. Influence of gender, menstrual phase, and oral contraceptive use on immunological changes in response to prolonged cycling. J. Appl. Physiol. 2005, 99, 979-985. [CrossRef]

37. Oosthuyse, T.; Bosch, A.N. The Effect of the Menstrual Cycle on Exercise Metabolism. Sports Med. 2010, 40, 207-227. [CrossRef]

38. Pedersen, B.K.; Febbraio, M.A. Muscle as an endocrine organ: Focus on muscle-derived interleukin-6. Physiol. Rev. 2008, 88, 1379-1406. [CrossRef]

39. Pedersen, B.K.; Fischer, C.P. Beneficial health effects of exercise-The role of IL-6 as a myokine. Trends Pharmacol. Sci. 2007, 28, 152-156. [CrossRef]

40. Pedersen, B.K. Anti-inflammatory effects of exercise: Role in diabetes and cardiovascular disease. Eur. J. Clin. Investig. 2017, 47,600-611. [CrossRef]

41. Liu, Z.; Que, S.; Xu, J.; Peng, T. Alanine aminotransferase-old biomarker and new concept: A review. Int. J. Med. Sci. 2014, 11, 925-935. [CrossRef] [PubMed]

42. Bekkelund, S.I.; Jorde, R. Alanine Aminotransferase and Body Composition in Obese Men and Women. Dis. Markers 2019, 2019, 1-9. [CrossRef] [PubMed]

43. Pal, S.; Chaki, B.; Chattopadhyay, S.; Bandyopadhyay, A. High-Intensity Exercise Induced Oxidative Stress and Skeletal Muscle Damage in Postpubertal Boys and Girls. J. Strength Cond. Res. 2018, 32, 1045-1052. [CrossRef] [PubMed]

44. Wang, I.L.; Hsiao, C.Y.; Li, Y.H.; Meng, F.B.; Huang, C.C.; Chen, Y.M. Nanobubbles Water Curcumin Extract Reduces Injury Risks on Drop Jumps in Women: A Pilot Study. Evid. Based Complement. Altern. Med. 2019, 2019. [CrossRef]

(C) 2020 by the authors. Licensee MDPI, Basel, Switzerland. This article is an open access article distributed under the terms and conditions of the Creative Commons Attribution (CC BY) license (http://creativecommons.org/licenses/by/4.0/). 